\section{EPV019/\#309 OVARIAN METASTASES FROM BREAST CANCER: A SERIES OF NINE CASES}

${ }^{1} \mathrm{~K}$ Ben Hamida*${ }^{*},{ }^{1,2}$ Zemni, ${ }^{3,4} \mathrm{~N}$ Boujelbene, ${ }^{5} \mathrm{~A}$ Mlouka, ${ }^{3} \mathrm{I}$ Zidi, ${ }^{5} \mathrm{~S}$ Essghaier, ${ }^{1} \mathrm{R}$ Chargui, ${ }^{1} \mathrm{~K}$ Rahal. ${ }^{1}$ Salah Azaiez Institute of Oncology, Surgical Oncology Department, Tunis, Tunisia; ${ }^{2}$ Sciences Faculty of Tunis, University of Tunis El Manar, Laboratory of Microorganismes and Active Biomolecules, Tunis, Tunisia; ${ }^{3}$ Sciences Faculty of Tunis, Laboratory of Microorganismes and Active Biomolecules, Tunis, Tunisia; ${ }^{4}$ Salah Azaiez Institute of Oncology, Pathology Department, Tunis, Tunisia; ${ }^{5}$ Salah Azaiez Institute of Oncology, Radiology Department, Tunis, Tunisia

\subsection{6/ijgc-2021-IGCS.86}

Objectives Development of ovarian metastasis (OM) during the course of primary breast cancer (PBC) is uncommon and associated with poor prognosis. The objective of this study is to review the characteristic clinical and imaging features of $\mathrm{OM}$ from PBC.

Methods A retrospective study of nine patients treated in our institution, who had documented OM from breast carcinoma between 2005 and 2020 .

Results At the time of PBC diagnosis, the mean age was 44 (range 31-64). In five cases, the PBC was unilateral. Histological subtypes were invasive ductal carcinoma in five patients and invasive lobular carcinoma in four patients. ER and PR were positive in eight cases. HER2 was positive in two cases. Five patients had locally advanced breast carcinoma. Four patients had synchronous ovarian metastases. Only three patients underwent breast surgery. Main symptoms of OM were pelvic pain and abdominal distension. An elevated CA 125 level was found in seven cases while CA 15-3 level was increased in eight cases. In four cases, pulmonary and bone metastases were simultaneously diagnosed with OM occurrence. All patients underwent ovarian surgery. Seven patients had bilateral OM. Ascites and peritoneal carcinomatosis were seen respectively in six and three patients. Time to occurrence of OM after PBC was a median of 25 months. The median follow-up period after OM assessment was 18 months.

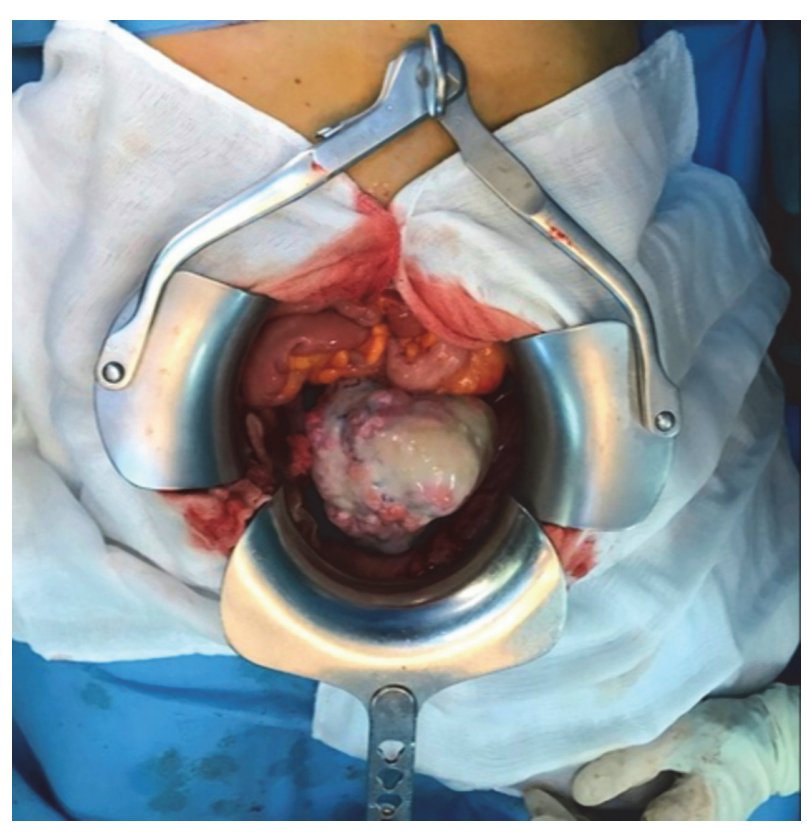

Abstract EPV019/\#309 Figure 1
Conclusions The evaluation of ovarian lesion years after breast cancer is challenging and rise the possibility of a metastatic lesion. Imaging, serum tumor markers and histology may provide valuable tools in the assessment of ambiguous cases.

\section{EPV020/\#311 SIGMOID COLON METASTASIS FROM MEDULLARY BREAST CARCINOMA MIMICKING PRIMARY SIGMOID COLON CANCER}

${ }^{1} \mathrm{H}$ Bouaziz, ${ }^{2} \mathrm{~N}$ Tounsi ${ }^{*},{ }^{2} \mathrm{~A}$ Jalleli, ${ }^{2} \mathrm{M}$ Ghalleb, ${ }^{3} \mathrm{~S}$ Kammoun, ${ }^{2} \mathrm{M}$ Slimane, ${ }^{2} \mathrm{~K}$ Rahal. ${ }^{1}$ Salah Azaiez Institute of Oncology, Surgical Oncology, Tunis, Tunisia; ${ }^{2}$ Salah Azaiez Institute of Oncology, Surgical Oncology, Tunis, Tunisia; ${ }^{3}$ Salah Azaiez Institute of Oncology, Department of Pathology, Tunis, Tunisia

\subsection{6/ijgc-2021-IGCS.87}

Objectives Solitary colorectal metastasis as the first and sole manifestation of spread is a rare occurrence and can be confused with primary intestinal malignancy. We reported our experience in management of sigmoid colon metastasis from medullary breast carcinoma.

Methods We presented here a case rarely reported in literature, showing sigmoid colonic metastasis from breast cancer.

Results A 64-year-old woman with a history of modified radical mastectomy (MRM), followed by adjuvant treatment, performed 19 years ago (2002) for medullar carcinoma in the right breast. She admitted to our hospital for abdominal pain and bowel obstruction syndrome. CT scan showed stenotic eccentric wall thickening of the distal sigmoid colon without metastatic lesion. A colostomy was realized in first time. Followed, secondarily by sigmoidectomy. The subsequent anatomopathological study and immunohistochemistry of the tumor showed metastasis of the carcinoma that was compatible with the primary breast carcinoma. PET-CT was requested and systemic chemotherapy was proposed.

Conclusions There is no consensus on the management of these uncommon lesions. Surgical treatment is reserved for cases of perforation, hemorrhage or intestinal obstruction.

\section{EPV021/\#383 ACCURACY OF PREDICT UK 2.1 IN PREDICTING SURVIVAL IN 'GRAY ZONE' RH+/HER2- BREAST CANCER: A POPULATION-BASED STUDY}

${ }^{1} \mathrm{D}$ Dhib, ${ }^{2} \mathrm{~N}$ Mejri, ${ }^{1} Y$ Berrazega*, ${ }^{1} \mathrm{H}$ Rachdi, ${ }^{1} \mathrm{H}$ Boussen. ${ }^{1}$ University hospital Abderrahmen Mami Ariana, Medical Oncology, Tunis, Tunisia; ${ }^{2}$ Abdrahman Mami Hospital Medical Oncology Department Tunisia, Medical Oncology, Ariana, Tunisia

\subsection{6/ijgc-2021-IGCS.88}

Objectives The aim of this study was to assess the validity of the online PREDICT tool in a population-based cohort of intermediate risk luminal breast cancer.

Methods Among the cohort of breast cancer patients $(n=962)$ treated between 2011-2017, 127 patients considered with intermediate risk $\mathrm{RH}+/$ HER2- tumors treated with adjuvant therapy were selected. Patients had at least one factor: $1-3 \mathrm{pN}$ ,$+>2 \mathrm{~cm}$, SBR II-III. Observed 5 -year overall survival were estimated using the Kaplan-Meier method, and compared with predicted outcomes using PREDICT UK 2.1, in the overall population and in several subgroups. 